\title{
Successful treatment of incidentally diagnosed retroperitoneal seminoma with primary external beam radiotherapy
}

\begin{abstract}
Background: Germ cell tumours (GCTs) are the most common cancer in young men, typically arising from the testes. However, a small subset (1\% to $2 \%$ ) appears to originate in other locations and is referred to as primary extragonadal germ cell tumors (EGCTs but origin remains controversial. New thought is that these so called primary retroperitoneal seminomas uniformly represent metastases from an occult or regressed testicular primary tumour.
\end{abstract}

Method: We present case report of retroperitoneal seminoma in a 60 year old male patient who presented with bloody diarrhea. Endoscopic visualization of colon was normal but imaging confirmed incidental finding of a nodal mass in the abdomen. Biopsy report confirmed classic seminoma. A gonadal primary was not seen on testicular ultrasound. Staging imaging and PET did not identify any other sites of disease.

Conclusion: Our case confirms the excellent response to radiation as monotherapy in retroperitoneal seminoma with no known primary. In order to understand this rare neoplasm and serve our patients well more emphasis should be on research for developing novel immunohistochemistry and markers.

Keywords: primary, retroperitoneal, seminoma, rare, burned out
Case Report

Volume 5 Issue 2 - 2018

\author{
Rashmi Koul,' Alshafa F,' Tai P,' Khoja L, ${ }^{3}$ \\ Czaykowski $\mathrm{P}^{4}$ \\ 'Department of Radiation Oncology, University of Manitoba, \\ Canada \\ ${ }^{2}$ Saskatchewan Cancer Agency, Canada \\ ${ }^{3}$ Medical student, Saudi Arabia \\ ${ }^{4}$ Department of Medical Oncology, University of Manitoba, \\ Canada
}

Correspondence: Rashmi Koul, Rashmi Koul, Department of Radiation Oncology, Cancercare Manitoba, 675 McDermot Ave, Winnipeg, MB. R3E0V9, Canada, Email rkoul@cancercare.mb.ca

Received: January 19, 2018 | Published: March 05, 2018

\section{Introduction}

Germ cell tumours (GCTs) in men are the most common neoplasm arising from the testes. However, a small subset ( $1 \%$ to $2 \%$ ) appear to originate in other locations and are referred to as primary extragonadal germ cell tumors (EGCTs). ${ }^{1}$ Those originating in the retroperitoneum represents only $30 \%-40 \%$ of all primary EGCTs. ${ }^{2}$ Most EGCTs (83\%) are of non-seminomatous histology as reported in the contemporary medical literature. ${ }^{3}$ Diagnosis of a primary retroperitoneal EGCT is usually made in later stages as compared to metastatic disease of a testicular origin due to the lack of a distinct, palpable testicular mass which typically triggers a patient to seek medical attention. ${ }^{4}$ The most common clinical manifestations of retroperitoneal EGCTs are vague abdominal and back pain. Other less frequent symptoms include weight loss, palpable abdominal mass, fever, dyspnea, and venous thrombosis. ${ }^{5}$ EGCT produce a wide variety of symptoms and may reach large volumes if they arise in "silent" areas. We report a rare case of a 60-year-old male diagnosed incidentally on a CT scan with retroperitoneal seminoma with no known primary. He was treated with curative radical radiotherapy.

\section{Case report}

A 60-year-old male with a previous history of haemorrhoids presented to his primary physician in June 2016 with bloody diarrhoea. He underwent colonoscopy and a computerized tomography (CT) scan of the abdomen and pelvis. The results of the colonoscopy were unremarkable. However, CT scan showed a few borderline enlarged lymph nodes in the retroperitoneum and at least three in the proximal left common iliac region with the largest node measuring up to $20 \mathrm{x}$ $11 \mathrm{~mm}$ (Figure 1). The nodes were interpreted as borderline enlarged and follow-up CT scan in six months was recommended. Repeat CT scan in January 2017 showed interval enlargement in one of the lymph nodes from $1.5 \times 1.6 \mathrm{~cm}$. to $2.2 \times 1.8 \mathrm{~cm}$ with the other nodes remaining stable (Figure 2). Due to the radiologic progression in one of the nodes, CT-guided core biopsies were obtained. They showed confluent clusters of large cells with vacuolated cytoplasm's and pleomorphic hyperchromatic nuclei with prominent one or more nucleoli. Scattered mitotic figures were seen. A prominent lymphocytic infiltrate was also present in the tissue. With immunohistochemistry, tumor cells were positive for CD117, OCT3/4, and podoplanin (D2-40). They also showed weak patchy positivity for placental alkaline phosphatase (PLAP). They were negative for cytokeratin markers, including broad spectrum cytokeratin (CK) (AE1/AE3), low molecular weight cytokeratin (Cam 5.2), CK7 and CK20. They were also negative for S100 protein and CD30. The morphologic and immunophenotypic features were in keeping with seminoma (Figure 3). The overall features, both histomorphologic and immunophenotypic were keeping with metastatic seminoma. On the basis of the biopsy, the patient had bilateral scrotal ultrasound which did not show any discreet testicular mass. The baseline tumor markers included beta HCG less than 1IU/L, LDH normal at 166 and alpha feto-protein of $4 \mathrm{ug} / \mathrm{L}$ and his remaining blood tests were completely normal. Physical examination was 
unremarkable. Patient had no past history of testicular maldescent Repeat CT scan of the chest, abdomen and pelvis in July 2017 was performed, which showed three left paraaortic masses, with the largest measuring up to $2.4 \times 2.4 \mathrm{~cm}$. There was no evidence of distant metastasis. The patient was diagnosed with stage II seminoma with no obvious testicular primary.

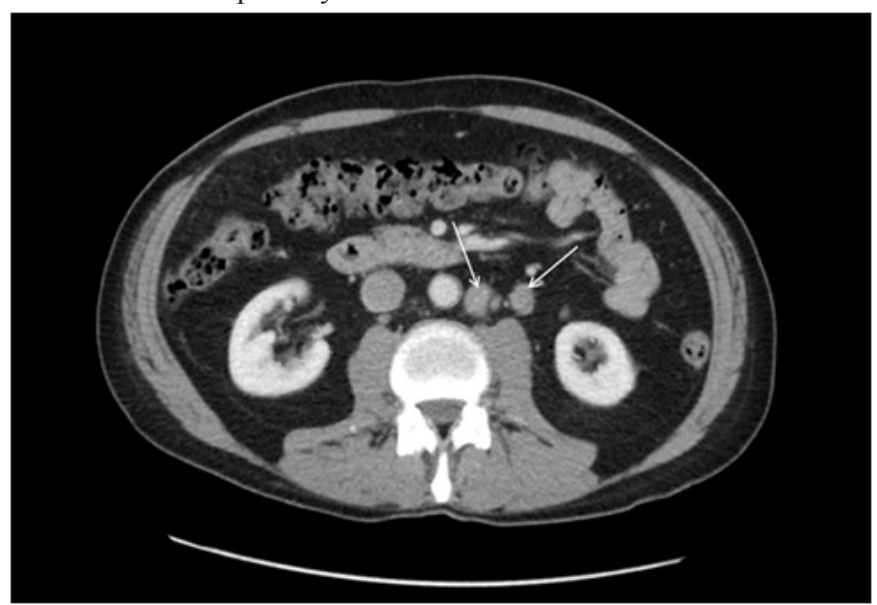

Figure I CT scan showed a few borderline enlarged lymph nodes in the retroperitoneum and at least three in the proximal left common iliac region with the largest node measuring up to $20 \times 1 \mathrm{Imm}$.

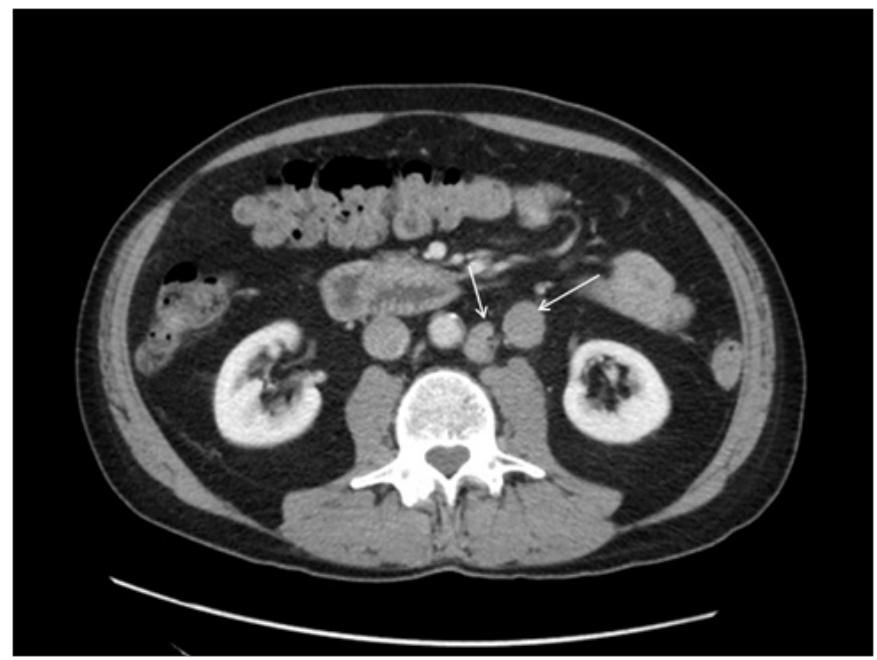

Figure 2 Repeat CT scan in January 2017 showed interval enlargement in one of the lymph nodes from $1.5 \times 1.6 \mathrm{~cm}$. to $2.2 \times 1.8 \mathrm{~cm}$ with the other nodes remaining stable.

A positron emission tomography (PET) scan was done in August 2017 which showed mild metabolic activity in a small right superior juglar lymph node with mildly active nodes inferior to this. These nodes were felt to be reactive in nature. No FDG uptake was seen in the thorax. Small pulmonary nodules seen on CT scan were below size limits for accurate evaluation. There was an intensely hypermetabolic enlarged left periaortic lymph node, standardized uptake values (SUV) 18, with smaller hypermetabolic nodes medial to this (Figure 4). These were at the level of the lower pole of the kidneys. The smaller node at the level of the aortic bifurcation did not have significant metabolic activity. No other metabolic activity was seen elsewhere. No abnormal activity was identified in the testes or the rest of the reproductive system. Therefore no other primary site was identified on PET scan. The final conclusion was that the two hypermetabolic lymph nodes seen in abdomen were neoplastic in nature related to the known seminoma but the small node in the right neck was reactive in nature. A gonadal primary tumour was not identified.

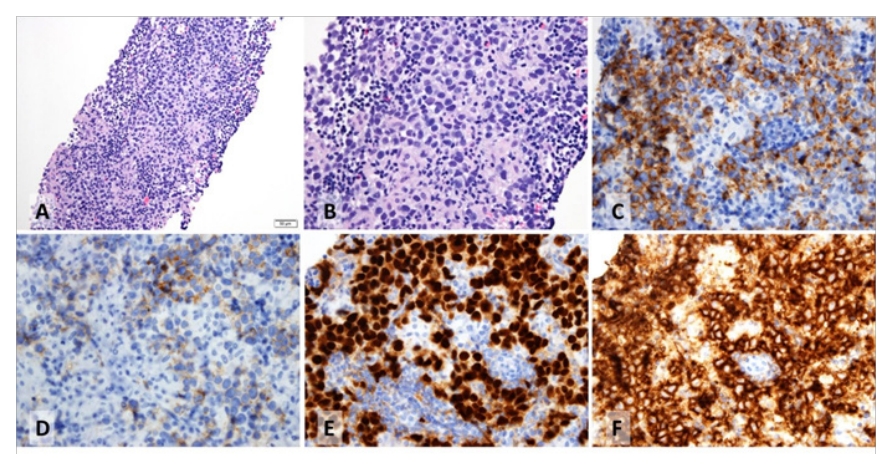

Figure 3 Histologic and immunophenotypic features of the tumor in one of the core biopsies. The tumor is composed of large cells with vacuolated cytoplasms with an intervening stroma that contains prominent small lymphocytes (A, B; hematoxylin and eosin). Tumor cells are positive for CDII7 (C) and weakly and variably positive for PLAP (D). They are also positive for OCT3/4 (E) and podoplanin (F).

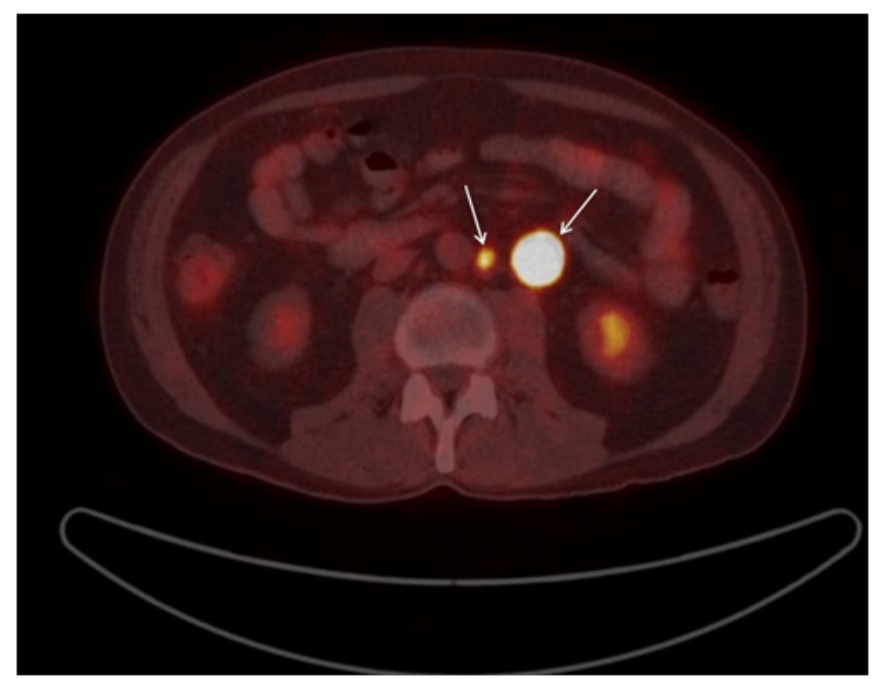

Figure 4 A positron emission tomography (PET) scan intensely hypermetabolic enlarged left periaortic lymph node, standardized uptake values (SUV) I8, with smaller hypermetabolic nodes medial to this.

The treatment options discussed with the patient included surgical resection of the nodal masses, chemotherapy or radiotherapy. The patient did not want to receive chemotherapy and decided to proceed with radical external beam radiation treatment. This case was discussed at multidisciplinary rounds and the consensus was to treat paraaortic, common iliac, both external and internal nodes bilaterally down to the top of obturator foramen. Radiation was planned as per nodal based anatomy. The nodal clinical target volume (CTV1) included the paracaval, precaval, inter-aortocaval, lateroaortic and preaortic lymph nodes. We contoured the inferior vena cava and aorta separately from $2 \mathrm{~cm}$ below the top of the kidneys down to the point where these blood vessels ended. A 1.2-cm expansion on the inferior vena cava and a larger, $1.9-\mathrm{cm}$ expansion on the aorta to include the lateroaortic nodes was added and edited to create a nodal CTV1. The 
bone and bowel were excluded from CTV1. A uniform 0.5-cm margin around was provided on CTV1 to create a planning target volume (PTV1) that accounted for treatment setup errors. A uniform $0.7-\mathrm{cm}$ margin around was provided on PTV1 to the block edge to take beam penumbra into account. This PTV1 received 20Gray in 10fractions, with 200centigray per fraction over two weeks (Figure 5). For the boost or cone down field, a CTV 2 was created around the PET avid nodes. PTV2 with a uniform $0.7-\mathrm{cm}$ margin to CTV 2 to the block edge was created (Figure 6). The organs at risk were charted out as below .The right and left kidney D50\% was 8 Gy (i.e., no more than $50 \%$ of the volume of each kidney should receive $8 \mathrm{~Gy}$ or higher). The mean dose to the right and left kidneys combined was less than 9Gy .For the boost, the right and left kidney D50\% was 2 Gy (i.e., no more than $50 \%$ of the volume of each kidney should receive $2 \mathrm{~Gy}$ or higher). This plan was different from typical dog-leg as we treated a Y-shaped radiation field given the fact we had no primary laterality of disease. The patient received a total dose of 36 Gray in 18 fractions (Figure 7). PTV1 and PTV2 coverage doses were equal to $95 \%$ and $107 \%$ of prescribed doses, respectively. The goal was to cover $100 \%$ of PTV1 and PTV2 with $95 \%$ of the prescribed dose. During the radiation treatment, the patient did not experience any gastrointestinal symptoms, and his CBC remained constantly normal. A follow up CT abdomen and pelvis was done two months post radiation treatment which showed significant treatment response of the retroperitoneal lymphadenopathy. The largest abdominal lymph node previously measuring $2.4 \times 2.4 \mathrm{~cm}$. has reduced in size to $1 \times 1 \mathrm{~cm}$. Also, no new retroperitoneal, pelvic or inguinal lymph nodes were identified (Figure 7). Six months post radiation PET scan showed no FDG uptake in the known nodal areas. This represents a complete response to radiation (Figure 8)

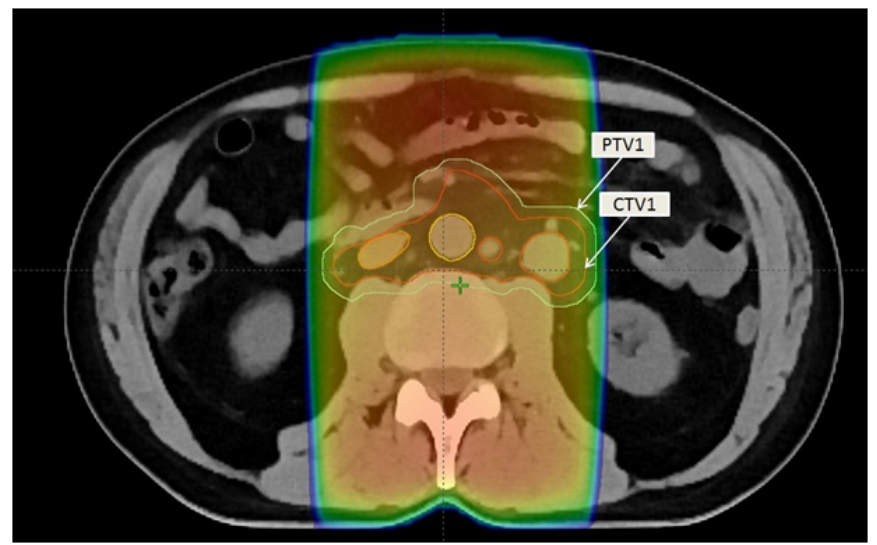

Figure 5 CT simulation image showing contours of clinical target volume (CTVI) and a uniform 0.5- $\mathrm{cm}$ margin around CTVI to create a planning target volume (PTVI).This PTVI received 20 Gray/ 10 fractions, with/200 centigray per fraction over two weeks.

\section{Discussion}

Primary retroperitoneal germ cell tumors are very uncommon. There are two main hypotheses in the literature explain the genesis of such neoplasms. The first hypothesis speculates that during embryogenesis, failure in the migration of germinal epithelium from the urogenital ridge to the scrotum may eventually lead to the establishment of germ cell tissue elsewhere in the body. ${ }^{6}$ The other hypothesis is that these tumors develop in germ cells normally distributed in midline locations throughout the body. ${ }^{7}$ However there is ongoing controversy in literature regarding whether primary extragonadal germ cell tumors really exist or not. It is a very old controversy that is alive and well documented in the literature. Generally speaking there are two dimensions to this controversy, one is theoretical and relates to the documented biology of germ cell neoplasia, while the other is more practical and relates to how one should practically investigate and manage a patient who is found to have a germ cell neoplasm. Old as well as newer papers in the literature most of which continue to question the existence of primary extragonadal germ cell tumors, especially in the retroperitoneum. ${ }^{8}$ Everyone should have very high skepticism that a germ cell tumor in the retroperitoneum (like the seminoma in our case) is primary, and that it should rather be considered metastatic until proven otherwise. A recent review from the International Society of Urological Pathology Testis Consultation Panel emphasizes "Although in the past some germ cell tumours have been labelled as 'primary' retroperitoneal tumours, current thinking is that these uniformly represent metastases from an occult or regressed testicular primary tumour. ${ }^{9}$

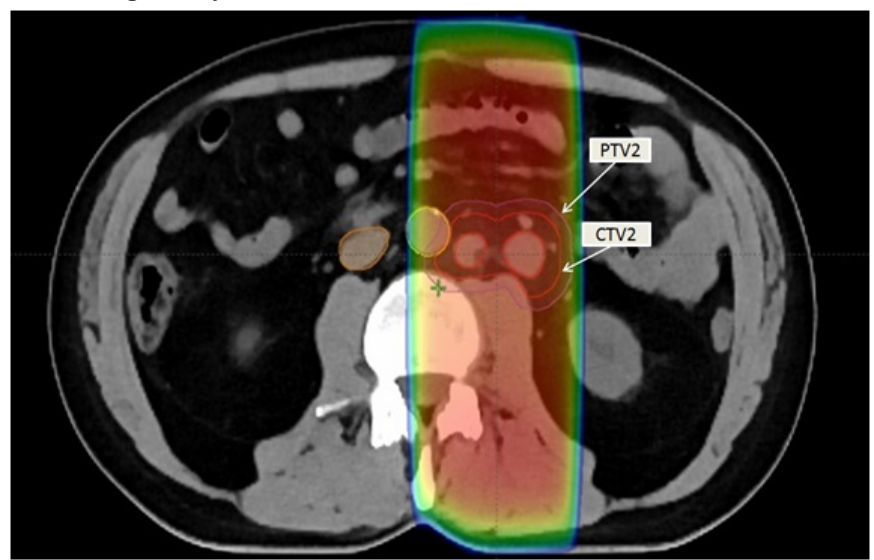

Figure $6 \mathrm{CT}$ simulation contouring images showing the boost field including CTV 2 and PTV2 as per PET avid nodes.

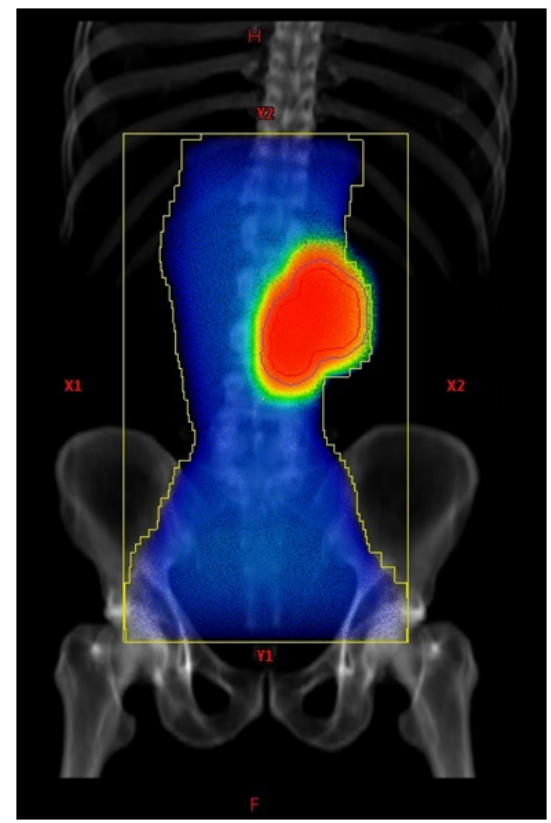

Figure 7 Y-shaped radiation field which received a total dose of 36 Gray in 18 fractions. 


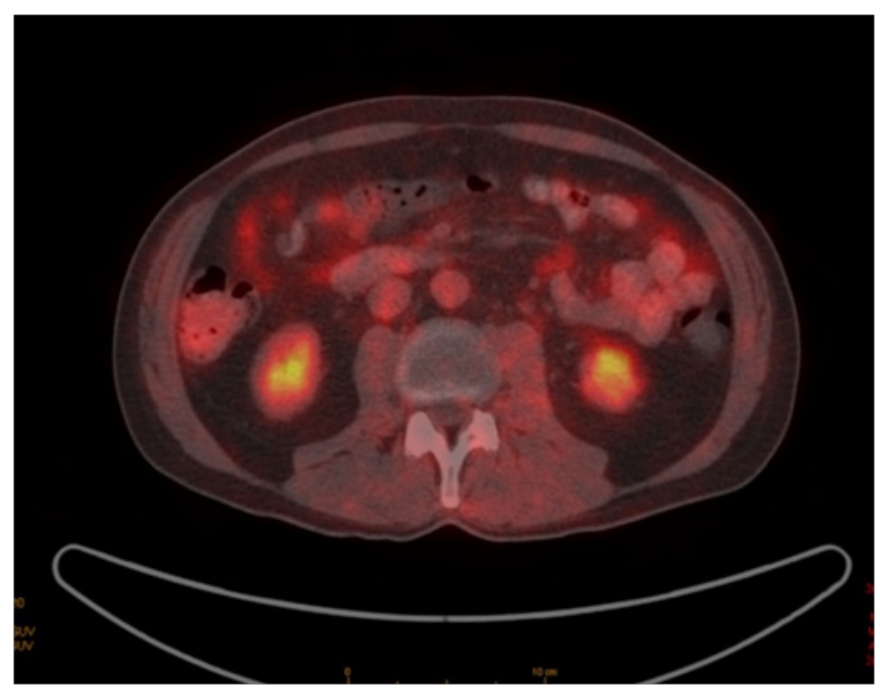

Figure 8 Post radiation PET scan showed no FDG uptake in the known noda areas.

A notable paper by Abell et al. ${ }^{10}$ documents 10 cases of primary retroperitoneum seminomas. They conclude that a retroperitoneal seminoma without any detectable or subsequent appearance of a tumor in either testis can be presumed to be primary, in one of three situations, namely 1) if gonadal tissue was found in its capsule, 2) if the lesion is encapsulated without lymph node involvement or 3) if the lesion is high in the retroperitoneum with adjacent lymph node involve ment but no involvement of the lower aortic, iliac or pelvic lymph nodes. ${ }^{10}$

Usually primary EGCTs are located in the midline; whereas metastatic tumors are located more laterally typically on the side of the primary testicular tumor. ${ }^{11}$ It seems in literature performing an ultrasound of the testes is an essential investigation to establish the diagnosis. ${ }^{12}$ Ultrasound findings are often normal in primary EGCT, which was the case in our patient. On the other hand, burnedout primary testicular tumors would show certain abnormalities on ultrasound, such as: echogenic foci, microlithiais, scar formation and inhomogeneity. ${ }^{13}$ Kirschling et al. ${ }^{14}$ concluded that high-resolution ultrasonography appears to be sufficient evidence to allow observation without surgical intervention. ${ }^{14}$ European consensus paper on the diagnosis and treatment of germ cell cancer the authors accepted the idea of a primary extragonadal germ cell tumor in a subset of patients (about a third of the cases who do not have intratubular germ cell neoplasia or ultrasonographic features that suggest a "burned-out" testicular primary). ${ }^{15}$ In theory, one would have to histologically examine both testes in an exhaustive manner to see if there is any evidence of current early, established or even regressed germ cell neoplasia, including "carcinoma in situ" (intratubular germ cell neoplasia or ITGCN, now called germ cell neoplasia in situ, or GCNIS by the WHO). ${ }^{16}$ One would also have to follow patients up for many years. In some cases a metachronous testicular germ cell tumor would become apparent years after the initial presentation. Furthermore, in addition to ultrasound of the testes, CT scan of the chest, abdomen and pelvis, PET-CT scan should be done to rule out an unknown primary tumor. Due to the rarity of this pathology, the advantage of PET-CT scans over CT in the initial diagnosis of a primary EGCT has not been established yet in the literature. With that being said, performing a
PET-CT scan can be appropriate, particularly in cases of seminoma, which typically have higher FDG uptake when compared to nonseminomatous tumor. ${ }^{17}$

Treating primary EGCTs is similar to treating germ cell tumors arising from the testes, except for the need to undergo an orchiectomy. Data from one of the largest EGCT series conducted has shown that in cases of retroperitoneal seminomas $(n=51)$, only $12 \%$ have undergone radiation therapy treatment alone. The reason behind this may be attributed to the fact that most of these tumors are diagnosed in later stages, necessitating chemotherapy. Median size of the primary retroperitoneal masses in their study was $7 \mathrm{~cm}^{2} .{ }^{18}$ Data from other studies have also shown primary tumor size retained prognostic importance and a scale of relapse risk based on the unit increment of tumor size was developed to help guide patients and clinicians in decision making. ${ }^{19}$ Fortunately, our patient had undergone a CT scan prior to the development of symptoms and was found to have three masses incidentally, with the largest measuring only $2.4 \mathrm{~cm}$.

Some of the studies have shown favorable outcomes both for short and long follow-up periods with combined modality treatment approach. ${ }^{20}$ One of the case series by Bokemeyer et al. ${ }^{21}$ in 2001 reports on six hundred thirty-five patients with extragonadal GCT who were treated consecutively at 11 centers in the United States and Europe during the cisplatin-based chemotherapy era between 1975 and 1996. Patients received chemotherapy with or without radiation therapy. No surgical resection was done in these patients. Follow up was almost 49 months. They reported 5 year overall survival of $88 \%$ in 52 patients with retroperitoneal seminoma in this series. Over all in patients with extragonadal seminoma, a survival rate of $>90 \%$ at 5 years is achieved with adequate cisplatin-based chemotherapy. Compared with patients with nonseminomatous extragonadal GCT, no difference in long term survival exists between patients with primary retroperitoneal or mediastinal seminoma location. Primary radiotherapy was associated with a significantly higher rate of disease recurrence, although most patients were salvaged by subsequent chemotherapy. ${ }^{21}$

Dr Einhorn ${ }^{22}$ reported the role of combination chemotherapy in disseminated testicular cancers in $1977 .{ }^{22}$ Subsequently many oncologists tried dose escalation chemotherapy with cisplatin and new regimens. In 1985 carboplatin replaced cisplatin in many trials due to reduced toxicity profile. ${ }^{23}$ All patients with germ cell pathology requiring chemotherapy currently receive treatment with cisplatinor carboplatin-based. ${ }^{24}$ Post chemotherapy surgery for advanced testicular cancer has evolved over the last couple of decades. Patients with nonseminomatous germ cell tumors and residual retroperitoneal mass $\geq 1 \mathrm{~cm}$ may undergo retroperitoneal lymph node dissection (RPLND). For seminoma, RPLND is considered in those patients with masses $\geq 3 \mathrm{~cm}$ that are also positron emission tomography positive. ${ }^{25} \mathrm{In}$ one study positive PET results correlated with the presence of viable tumor, but patients with residual mass and negative PET findings still required further treatment most commonly resection. In patients who had disease progression seen on imaging or increased levels of tumour markers additional PET imaging had no benefit. PET seems useful in patients with stable disease or partial remission in imaging. ${ }^{26}$

Radiation remains one of the preferred treatment approaches for early stage seminoma even in the modern era. ${ }^{27}$ Traditionally, radiation portals encompassed the para-aortic, ipsilateral iliac and obturator nodes in a 'dog-leg' (DL) field to a dose of 30Gy. Although local control rates were excellent, long term data provided concern 
regarding late sequelae. ${ }^{28}$ The basic intent for classic seminoma is to target the lymphatic pathways draining the testicles. Groups have mapped the location of para-aortic, paracaval, interaortocaval, preaortic, and iliac lymph nodes relative to major blood vessels. As per mapping $90 \%$ of para-aortic, paracaval, interaortocaval, and preaorticlymph nodes in $90 \%$ of patients will be encompassed by symmetrical, three-dimensional expansion of the inferior vena cava by $1.2 \mathrm{~cm}$. The CTV should be trimmed to exclude bone, bowel, muscle, fascia and bladder. The use of vascular rather than bony anatomy ensures a high probability of encompassing most of the nodal tissue at risk of harboring metastases in most patients while minimizing the radiation dose delivered to normal tissues. ${ }^{29}$ In our case we chose to treat lymphatics draining both sides of the retroperitoneum as no laterality was established in the testicles clinically or by imaging.

\section{Conclusion}

Primary retroperitoneal seminoma is an entity rarely encountered in our practice. Differentiating a primary EGCT from metastatic adenopathy arising from an occult testicular primary can be quite difficult. The new thought is that most likely these so called primary retroperitoneal seminomas uniformly represent metastases from an occult or regressed testicular primary tumour. Longer follow up is recommended. Radiation and/ or chemotherapy can be curative and yield good outcomes.

\section{Acknowledgements}

None.

\section{Conflict of interest}

The author declares no conflict of interest.

\section{References}

1. Walsh PC, Retik AB, Darracott VE, et al. Neoplasm of the testis. Campbell's Urology. 8th ed. London: WB Saunders; 2002.

2. Albany C, Einhorn LH. Extragonadal germ cell tumors: Clinical presentation and management. Curr Opin Oncol. 2013;25(3):261-265.

3. Bokemeyer C, Nichols CR, Droz JP, et al. Extragonadal germ cell tumors of the mediastinum and retroperitoneum: Results from an international analysis. J Clin Oncol. 2002;20(7):1864-1873.

4. Siegel RL, Miller KD, Jemal A. Cancer statistics. CA Cancer J Clin. 2016;66(1):7-30

5. Borrego Hernándo J, Guinda Sevillano C, Laguna Pes MP, et al. Retroperitoneal germinal tumor: extragonadal or burned out phenomenon of testicular primary? Presentation of a clinical case. Actas Urol. 1998;22(2):170-174.

6. Einhorn LH, Richie JP, Shipley WU. Extragonadal germ cell tumors. In: Cancer Principles and Practice of Oncology. Philadelphia: Lippincott; 1993.

7. Friedman NB. The function of primordial germ cell in extragonadal tissue. Int J Androl. 1987;10(1):43-96.

8. Scholz M, Zehender N, Borner H, et al. Extragonadal retroperitoneal germ cell tumor: evidence of origin in the testis. Annals of Oncology. 2002;13(1):121-124.

9. Williamson SR, Delahunt B, Magi-Galluzzi C, et al. The World Health Organization 2016 classification of testicular germ cell tumours: a review and update from the International Society of Urological Pathology Testis Consultation Panel. Histopathology. 2017;70(3):335346.

10. Abell MR, Fayos JV, Lampe I. Retroperitoneal germinomas without evidence of testicular involvement. Cancer. 1965;18:273-290.

11. Kozłowski P, Starosławska E, Szumiło J, et al. Epidemiology and risk factors of testicular tumours. Pol Merkur Lekarski. 2016;40(238):211215.

12. Bockrath JM, Schaeffer AJ, Kies MS, et al. Ultrasound identification of impalpable testicle tumor. J Urol. 1983;130(2): 355-356.

13. Yee WS, Kim YS, Kim SJ, et al. Testicular microlithiasis: prevalence and clinical significance in a population referred for scrotal ultrasonography. Korean J Urol. 2011;52(3):172-177.

14. Kirschling RJ, Kvols LK, Charboneau JW, et al. High-resolution ultrasonographic and pathologic abnormalities of germ cell tumors in patients with clinically normal testes. Mayo Clin Proc 1983;58(10):648653.

15. Krege S, Beyer J, Souchon R, et al. European Consensus Conference on Diagnosis and Treatment of Germ Cell Cancer: A Report of the Second Meeting of the European Germ Cell Cancer Consensus group (EGCCCG): Part I. Eur Urol 2008;53(3):478-496.

16. Dieckmann KP, Skakkebaek NE. Carcinoma in situ of the testis: review of biological and clinical features. Int J Cancer 1999;83(6):815-822.

17. Cremerius U, Effert PJ, Adam G, et al. FDG PET for detection and therapy control of metastatic germ cell tumour. $\mathrm{J} \mathrm{Nucl} \mathrm{Med}$. 1998;39(5):815-822.

18. Shinagare AB, Jagannathan JP, Ramaiya NH, et al. Adul extragonadal germ cell tumors. American Journal of Roentgenology. 2010;195(4):W274-280.

19. Chung P, Daugaard G, Tyldesley S, et al. Evaluation of a prognostic model for risk of relapse in stage I seminoma surveillance. Cancer Med. 2015;4(1):155-160.

20. Bokemayer C, Droz JP, Horwich A, et al. Extragonadal seminoma: an international multicenter analysis of prognostic factors and long term treatment outcome. Cancer. 2001;91(7):1394-1401.

21. Einhorn LH, Donohue JP. Cisplatin, Vinblastine and Bleomycin combination chemotherapy in disseminated testicular cancer. Ann Int Med 1977;87(3):293-298.

22. Horwich A, Oliver RT, Wilkinson PM, et al. A medical research council randomized trial of single agent carboplatin versus etoposide and cisplatin for advanced metastatic seminoma: MRC Testicular Tumour Working Party. Br J Cancer. 2000;83(12):1623-1629.

23. Donohue JP, Foster RS. Integration of surgery and systemic chemotherapy: results and principles of integration. Semin Urol Oncol. 1998;16(2):65-71.

24. Toner GC, Panicek DM, Heelan RT, et al. Adjunctive surgery after chemotherapy for nonseminomatous germ cell tumors: recommendations for patient selection. J Clin Oncol. 1990;8(10):16831694

25. Pfannenberg AC, Oechsle K, Bokemeyer C, et al. The role of [(18) F] FDG-PET, CT/MRI and tumor marker kinetics in the evaluation of post chemotherapy residual masses in metastatic germ cell tumors-prospects for management. World J Urol. 2004;22(2):132-139.

26. Dong W, Gang W, Liu M, et al. Analysis of the Prognosis of Patients with Testicular Seminoma. Oncol Lett. 2016;11(2):1361-1366. 
27. Jonska-Gmyrek J, Peczkowski P, Michalski W, et al. Radiotherapy in Testicular germ cell Tumours- a literature review. Contemp Oncol 2017;21(3):203-208
28. Wilder Richard B, Mark K. Buyyounouski, Jason A Efstathiou, et al. Radiotherapy Treatment Planning for Testicular Seminoma. International Journal of Radiation Oncology. 2012;83(4):445-452. 